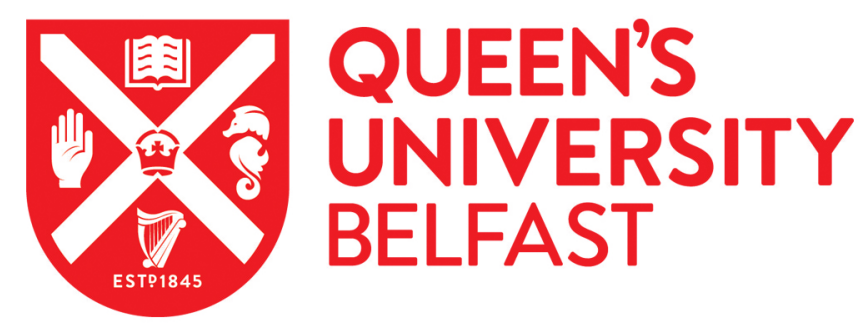

\title{
Fasciola gigantica: Comparison of the tegumental ultrastructure in newly excysted metacercariae and in vitro penetrated juvenile flukes indicates intracellular sources of molecules with vaccinal and immunomodulatory potential
}

Hanna, R. E. B., Moffett, D., Robinson, M. W., Jura, W. G. Z. O., Brennan, G. P., \& Fairweather, I. (2019). Fasciola gigantica: Comparison of the tegumental ultrastructure in newly excysted metacercariae and in vitro penetrated juvenile flukes indicates intracellular sources of molecules with vaccinal and immunomodulatory potential. Veterinary Parasitology, 265, 38-47. https://doi.org/10.1016/j.vetpar.2018.11.019

Published in:

Veterinary Parasitology

Document Version:

Peer reviewed version

Queen's University Belfast - Research Portal:

Link to publication record in Queen's University Belfast Research Portal

Publisher rights

(C) 2018 Elsevier Ltd.

This manuscript version is made available under the CC-BY-NC-ND 4.0 license http://creativecommons.org/licenses/by-nc-nd/4.0/,which permits distribution and reproduction for noncommercial purposes, provided the author and source are cited.

\section{General rights}

Copyright for the publications made accessible via the Queen's University Belfast Research Portal is retained by the author(s) and / or other copyright owners and it is a condition of accessing these publications that users recognise and abide by the legal requirements associated with these rights.

Take down policy

The Research Portal is Queen's institutional repository that provides access to Queen's research output. Every effort has been made to ensure that content in the Research Portal does not infringe any person's rights, or applicable UK laws. If you discover content in the Research Portal that you believe breaches copyright or violates any law, please contact openaccess@qub.ac.uk. 
Fasciola gigantica: comparison of the tegumental ultrastructure in newly excysted metacercariae and in vitro penetrated juvenile flukes indicates intracellular sources of molecules with vaccinal and immunomodulatory potential

R.E.B. Hanna a, ${ }^{a}$, D. Moffett ${ }^{a}$, M. W. Robinson ${ }^{\text {b }}$, W.G.Z.O. Jura ${ }^{\text {c }, ~ G . P . ~ B r e n n a n ~}{ }^{b}$, I. Fairweather $^{\text {b }}$

a Veterinary Sciences Division, Agri-Food and Biosciences Institute (AFBI), Stormont, Belfast BT4 3SD, United Kingdom

${ }^{b}$ School of Biological Sciences, The Queen's University of Belfast, Belfast BT9 7BL, United Kingdom

' Department of Zoology, Maseno University, P.O. Box 333-40105, Maseno, Kenya

\begin{abstract}
Using in vitro procedures to prepare newly excysted metacercariae and gut-penetrated juvenile Fasciola gigantica, the ultrastructural features of the tegumental syncytium and perikarya of these ephemeral stages in the host-invasion process were compared. The T0type tegumental cells in newly excysted metacercariae are packed with stored T0 granules which, following transport to the surface membrane of the syncytium, discharge by exocytosis to maintain the glycocalyx. The T0 cells become depleted of T0 granules during the penetration process, shrink in size, and initiate autophagy in the cytoplasm to facilitate metamorphosis from a storage function to active biosynthesis. The novel products appear to include lysosomes which contribute to the autophagosomes, and T1 granules, necessary for maintenance of the glycocalyx and immunoprotection, as the invasion process continues into the host liver. Residual bodies, the end-products of autophagy, are eliminated from the apical membrane of the tegumental syncytium into the host-parasite interface. There they may present a transient source of parasite-derived molecules, including lysosomal cathepsin-type proteases, with potential for interaction with the host's immune system, and so might be exploited as targets for vaccinal and immunomodulatory studies.
\end{abstract}

Key words: Fasciola gigantica; in vitro excystment and gut-penetration; tegumental ultrastructure; glycocalyx; autophagy; vaccine target molecules. 


\section{Introduction}

Fasciolosis caused by Fasciola gigantica infection is regarded as among the most important helminth diseases of ruminants in Asia and Africa, representing a significant factor in limiting livestock production that may impact on global food production, as demand for meat production increases in developing countries (Spithill et al., 1999). Despite its worldwide significance, little is known of the molecular biology and immunogenicity of $F$. gigantica, in comparison to Fasciola hepatica, likely reflecting the predominance of the former in developing countries with limited financial resources for basic studies on ruminants. It is becoming clear that the often-assumed similarity between these two species may be an over-simplification. Recent genomic studies have revealed that, not only are the two sister species well separated by mitochondrial and nuclear genome sequences, but African and Asian F. gigantica populations are sufficiently separated to be considered as distinct species, while European $F$. hepatica-like fluke and African high altitude $F$. hepaticalike fluke may also constitute distinct species (Walker et al., 2012). Clearly this genetic divergence between morphologically similar populations of Fasciola may well be reflected at the proteome level. In particular, the expression of potentially immunogenic molecules at the host-parasite interface, or in parasite excretions/secretions, may well differ fundamentally between species, populations and indeed life-cycle stages of the various clades. Although much research into vaccination against Fasciola spp. has been undertaken, no commercial vaccine is as yet available, but certain cysteine proteases of the cathepsin family in adult fluke and metacercariae of $F$. hepatica and $F$. gigantica appear to be promising targets (Jayaraj et al., 2010; Fairweather, 2011). Cathepsins also feature amongst the many molecules secreted by $F$. hepatica that interact with host immune cells such as dendritic cells, macrophages and mast cells and direct the immune response towards a favourable non-protective Th2-mediated/regulatory environment (Dalton et al., 2013). Enzymes of this group are activated by the low pH found in lysosomes, and their activity is often associated with these organelles, although some function extracellularly, after secretion from lysosome-like bodies (Turk et al., 2012). Amongst the earliest-appearing and most potent immunogenic components of $F$. hepatica are those associated with the tegumental glycocalyx, which is actively sloughed from the surface membrane at the hostparasite interface, and is replaced by exocrine secretion of GER-Golgi-derived 'TO' and 'T1'secretory granules (Bennett and Threadgold, 1975; Hanna, 1980 a, b; Hanna and Trudgett, 1983). While the tegumental glycocalyx also appears to provide the earliest antigenic stimulus for the bovine host's immune response to F. gigantica (Hanna and Jura, 1977), the ultrastructural correlates of glycocalyx production have not been documented for this fluke species. The aim of the present study is to examine the morphology of the tegument in those life-cycle stages of $F$. gigantica that represent the first encounter of parasite with host. These are the fluke stages that present the host with the earliest opportunities for immune recognition and response, while needing to protect themselves in a novel and challenging physiological and immunological environment. The identification of ultrastructural features and organelles associated with tegumental biosynthetic and secretory activity may highlight targets in the search for putative vaccine and immunomodulatory candidates. 


\section{Methods and materials}

\section{General}

The preparative stages of this study, namely collection, excystment, penetration, fixation and embedding of $F$. gigantica larvae for electron microscopy were carried out in the (then) East African Veterinary Research Organisation, Muguga, Kenya, in 1975. Subsequent sectioning and examination of the material was carried out at the Veterinary Sciences Division, Agri-Food and Biosciences Institute, Belfast, in 2018.

\section{Source of material}

Metacercarial cysts were obtained from a long-standing colony of Lymnaea natalensis maintained in the Parasitology Department of the East African Veterinary Research Organisation, Muguga, Kenya. Originally, snails in this laboratory colony had been infected with miracidia hatched from eggs that had been derived from $F$. gigantica individuals collected from cattle at Athi River abattoir, Nairobi, Kenya. These 'parent' flukes used for egg collection had been visually confirmed to be $F$. gigantica on the basis of gross morphology. Cercariae, shed 6 weeks or more after infection of snails, were allowed to encyst on grass blades and were subsequently stored at $4^{\circ} \mathrm{C}$ before use.

\section{Excystment}

Metacercarial cysts were dissected free from their fibrous, cup-shaped outer coats by applying gentle pressure with a fine glass needle. Non-viable cysts, which had a cloudy homogenous appearance, were discarded at this stage. In vitro excystment was carried out by a method based on that of Dixon (1964) for F. hepatica. Viable cysts were immersed in excystment fluid which was prepared immediately before use by mixing equal volumes of two solutions ( $A$ and $B$ ) and adding L-cysteine hydrochloride crystals $(0.2 \% \mathrm{w} / \mathrm{v}$ of working solution). Solution A was $0.5 \mathrm{M} \mathrm{HCl}$ and Solution B was an aqueous solution of $\mathrm{NaHCO} 3(1 \%$ $\mathrm{w} / \mathrm{v}), \mathrm{NaCl}(0.8 \% \mathrm{w} / \mathrm{v})$ and whole filtered ox bile $(20 \% \mathrm{v} / \mathrm{v})$, which was stored at $4^{\circ} \mathrm{C}$. Incubation was at $37^{\circ} \mathrm{C}$ for $2 \mathrm{~h}$, following which the excysted metacercariae were pipetted into $\mathrm{NaCl}$ solution $(0.8 \% \mathrm{w} / \mathrm{v})$ for rinsing at $37^{\circ} \mathrm{C}$.

\section{Penetration}

Newly hatched larvae were suspended in Eagle's MEM medium (with streptomycin sulphate, $0.2 \% \mathrm{w} / \mathrm{v}$ and sodium benzyl-penicillin, $1 \mathrm{~m} \mathrm{iu} / \mathrm{l}$ ) at $37^{\circ} \mathrm{C}$. A length of mouse gut (duodenum and proximal jejunum) was removed from an animal freshly killed by cervical dislocation, and was flushed out with Eagle's MEM plus antibiotics. One end was ligated with cotton thread, and approximately 50 newly excysted metacercariae, suspended in medium, were introduced at the open end with a blunt glass Pasteur pipette. The gut 'bag' was closed by a second ligature and suspended in $25 \mathrm{ml}$ of Eagle's medium with antibiotics. The preparation was then incubated for $5 \mathrm{~h}$ at $37^{\circ} \mathrm{C}$. Following incubation, the medium was decanted and the larvae that had penetrated the gut (approximately 40 ) were collected and pipetted into fresh Eagle's medium with antibiotics at $37^{\circ} \mathrm{C}$. The larvae were then pipetted into $\mathrm{NaCl}$ solution $(0.8 \% \mathrm{w} / \mathrm{v})$ for rinsing at $37^{\circ} \mathrm{C}$. 
Batches of newly excysted metacercariae and newly penetrated juveniles were fixed by removing the saline solution in which they were held at $37^{\circ}$, and replacing this with $4 \%$ Millonig phosphate-buffered glutaraldehyde containing $3 \% \mathrm{w} / \mathrm{v}$ sucrose. After $24 \mathrm{~h}$ fixation at $4^{\circ} \mathrm{C}$, the larvae were washed thoroughly with Millonig buffer containing $3 \% \mathrm{w} / \mathrm{v}$ sucrose, post-fixed with $1 \% \mathrm{w} / \mathrm{v}$ Millonig-buffered osmium tetroxide, dehydrated through alcohol and propylene oxide, infiltrated and embedded in Araldite embedding resin (then supplied by Ciba-Geigy; currently supplied by Agar Scientific Ltd., Essex, UK), following the manufacturer's recommended protocol. Ultrathin sections (60-70 nm thick) were cut from the resin blocks using a Leica EM UC7 ultramicrotome, mounted on uncoated nickel grids, double stained with uranyl acetate and lead citrate and viewed in a JEOL JEM-1400 transmission electron microscope with an AMT Activue XR16 digital camera system, operating at an accelerating voltage of $80 \mathrm{kV}$.

\section{Results}

Newly excysted metacercariae.

Ten newly excysted metacercariae were examined. All showed similar morphological features in the tegument and in all other organs and systems. The tegument comprises an anucleate cytoplasmic surface syncytium, approximately $2 \mu \mathrm{m}$ in thickness, delimited by an irregular, deeply invaginated apical plasma membrane and an undulating basal membrane. The surface syncytium is connected by numerous irregular, tortuous, often vacuolated cytoplasmic tubules or 'trabeculae' to a nucleated zone (perikarya or 'tegumental cells') which is located beneath the myofibrils of the superficial circular and longitudinal muscles (Fig. 1a). The myofibrils themselves lie immediately beneath the basal plasma membrane and basal lamina of the tegumental syncytium, and are enveloped by a layer of interstitial material, through which the tegumental trabeculae penetrate. Within the cytoplasm of the syncytium, numerous large conical spines are present (Fig. 1b). These display a characteristic striated appearance (Fig. 1c), and are set at an angle to the basal membrane of the syncytium, their tips pointing posteriad, their full length retained within the syncytium, with the apical plasma membrane continuous over the tip. The base of each spine is marked by a dense junction with the basal plasma membrane of the syncytium. The basal lamina lies directly beneath (Fig. $1 \mathrm{~b}$ and c). Also within the syncytial cytoplasm are dense mitochondria (each featuring a few cristae), and fairly numerous oval, membrane-bound secretory granules (each approximately $160 \times 90 \mathrm{~nm}$ ) containing homogenous moderately electrondense material (hereafter referred to as 'TO' granules, Fig. 1b). At higher magnification, these are sometimes seen close to the apical membrane, where occasionally the content is seen on the external aspect of the membrane, leaving the limiting membrane, now collapsed as a flattened disc, within the syncytial cytoplasm (Fig. 2a). These flattened membranous discs are particularly numerous in the distal cytoplasm of the syncytium, while intact secretory granules predominate deeper in the cytoplasm (Fig. 2a and b). The outer aspect of the apical membrane features an irregular dense zone, giving the apical membrane a 'fuzzy' appearance (Fig. 2a). 
In the nucleated zone of the tegument, lying beneath the musculature, each tegumental cell contains a single large, generally ovoid, heterochromatic nucleus, with surrounding cytoplasm. The predominant type of organelle present in the cytoplasm is an ovoid secretory granule similar in all respects to those seen in the surface syncytium. These granules are seen densely packed in the cytoplasm, usually leaving only a thin zone of cytoplasm close to the nucleus and at the periphery of the cell (Fig. 1a and b). Within the cytoplasm, numerous ribosomes and polyribosomes are present, together with occasional strands of granular endoplasmic reticulum (GER) (Fig. 2c and d). Golgi fields, featuring stacked lamellae, are also present within the cytoplasm of the tegumental cells, and electron dense spherical secretory granules $80 \mathrm{~nm}( \pm 10 \mathrm{~nm})$ in diameter are often seen close to the maturing face (hereafter referred to as 'T1' granules, Fig. $2 \mathrm{c}$ and d). Dense mitochondria are abundant and similar to those seen in the surface syncytium (Fig. 2c and d). Irregularly-sized spherical lipid globules are also present in the tegumental cell cytoplasm (Fig. 2d). Occasionally, non-membrane-bound masses of striated material similar to that comprising the surface spines are seen in the tegumental cells (Fig. 3a), and rarely profiles of scroll-like bodies can be recognised (Fig. 3b). Each tegumental cell body is irregular, with numerous pseudopod-like protuberances of cytoplasm ramifying amongst projections of the muscle cells and parenchymal cells which also occupy the sub-syncytial zone. Where projections of the tegumental cells are juxtaposed to parenchyma and muscle cells and indeed other tegumental cell extensions, dense membrane junctions are frequently seen (Fig. 3c). The trabeculae are cytoplasmic extensions of the tegumental cells which meander towards the surface syncytium, eventually connecting with the basal membrane. Often the cytoplasm of the trabeculae is seen to contain numerous T0 granules, but at the junction with the basal membrane, there is usually a vacuolated space (Fig. 3d).

In vitro penetrated juvenile flukes.

Of approximately 50 newly excysted juveniles pipetted into each mouse gut bag, approximately 40 successfully penetrated to the suspending medium, consistent with previous findings (Hanna et al., 1975). Ten juvenile flukes, fixed after having penetrated through mouse gut bags in vitro, were examined. All exhibited similar morphological features in the tegument and in all other organs and systems. The most striking difference between these and the newly excysted metacercariae is the presence of numerous large but irregularly-sized, generally spheroidal to elliptical electron-dense bodies lying in the cytoplasm of the surface syncytium and tegumental cells (Fig. 3e). In the syncytium, these bodies are often seen close to the apical membrane, where they bulge from the surface, invested in a thin layer of cytoplasm, and apparently bud off from the surface by a form of apocrine secretion. This activity occurs over all parts of the fluke body, including the syncytial lining of the acetabulum (Fig. 3e). In the syncytium, T0 secretory granules are particularly abundant, especially in the apical cytoplasm, and spines are distributed similarly to those in the newly excysted metacercariae (Fig. 4a). The mitochondria sometimes appear swollen, and the matrix is less dense than in newly excysted metacercariae (Fig. $4 \mathrm{a}$ and b). In some sections, and in some areas of the body, dense T1 secretory granules, approximately 
$80 \mathrm{~nm}$ in diameter, appear in the syncytial cytoplasm (Fig. 4b), but the distribution of these is irregular and patchy. As in the newly excysted metacercariae, flattened membranous sacs, apparently representing empty T0 granules, are frequently seen below the apical plasma membrane, which has a generally 'fuzzy' appearance (Fig. 4b). Beneath the base of the syncytium, attached to the basal plasma membrane and basal lamina by junctional complexes (sometimes appearing as septate desmosomes), swollen cytoplasmic bulb-like structures are sometimes seen (Fig. 4c). These contain small vesicles, together with larger irregularly-sized spheroidal bodies of moderate electron density. Similar bulb-like structures containing variously sized and shaped membrane-bound bodies are also associated with the syncytium of newly excysted metacercariae (Fig. 2b).

In the nucleated zone of the tegument of in vitro penetrated juvenile flukes, T0 granules are much reduced in number, in comparison to the situation in newly excysted metacercariae. The tegumental cells themselves are shrunken and contracted, with a correspondingly large increase in electron-lucid space surrounding them, representing distention and vacuolation of ramifications of the parenchymal cells and muscle cells (Fig. 3e and 4d), to which the tegumental cell membranes are linked by dense junctions. Within the tegumental cell cytoplasm, the remaining T0 granules are often clustered and partially sequestered by membranes and vacuolated membranous sacs (Fig. 5a). In these areas, large irregular electron-dense bodies, similar to those seen in the surface syncytium, are in close proximity with the T0 granules, often incorporating and engulfing them (Fig. 5a, b and C). Large and smaller dense bodies are often seen to merge (Fig. 5d). High magnification of the periphery of the dense bodies reveals them to be membrane-bound (Fig. 6a), often with duplication and densification of the bounding layer (Fig. $6 \mathrm{~b}$ and C), and flattened dense membranous lamellae are sometimes seen apposing the periphery of the dense bodies (Fig. 6c). In the cytoplasm of the tegumental cells, ribosomes, polyribosomes and GER are well-represented, especially close to the nucleus (Fig. $5 \mathrm{~d}$ ). Stacked Golgi lamellae are not often seen, but Golgi fields are represented by vesicles of varying size and density, probably representing primary lysosomes, in the vicinity of the large dense bodies (Fig. $5 \mathrm{c}$ and d). Mitochondria appear dense with few cristae (Fig. 5d) or sometimes swollen and relatively electron-lucid (Fig. 5a). Scroll-like bodies are seen rarely (Fig. 6d).

\section{Discussion}

The experimental methods employed in this study to obtain newly excysted metacercariae and penetrated juvenile $F$. gigantica were described by Hanna et al. $(1975,1976)$ and Hanna and Jura (1976). Clearly, the collection of larvae at these early stages of development from infected host animals would have been time-consuming and relatively unproductive in terms of parasite recovery, so the in vitro methods were designed to allow rapid and efficient accumulation of large numbers of clean larvae for use in further work, including preparation for electron microscopy. The efficacy of the techniques in yielding undamaged and physiologically intact infectious stages was established by injecting newly excysted metacercariae, newly penetrated juvenile flukes, and in vitro-maintained juveniles into the peritoneal cavity of experimental mice, and comparing the establishment and growth rates 
achieved with those for infections initiated by oral gavage of metacercarial cysts. In fact, newly excysted or newly penetrated fluke administered intraperitoneally gave establishment rates significantly higher than metacercariae administered orally, and the length of the resulting flukes in mice at 4 weeks post-infection was not significantly different from that of 4-week-old flukes from cattle (Hanna and Jura, 1976).

The fine structure of the tegument in newly excysted $F$. hepatica was described by Bennett and Threadgold (1973), and early changes in its structure during migration through the peritoneal cavity and penetration of the liver in mice were detailed by Bennett and Threadgold (1975). While the tegument is organised as in the adult fluke (Threadgold, 1963, 1967), with a surface syncytium containing spines and secretory granules communicating with perinuclear regions below the muscle layers by means of connecting trabeculae, the newly excysted and early migrating stages have only one type of perinuclear region and one type of secretory granule, respectively termed 'TO tegumental cells' and 'TO secretory granules' by Bennett and Threadgold (1975), to distinguish them from the 'T1' and 'T2' tegumental cells and granules found in adult flukes and described by Threadgold (1963, 1967). In the present study, newly excysted $F$. gigantica metacercariae were, like $F$. hepatica, found to contain one type of perinuclear region and one type of secretory granule, herein designated 'TO'. However the TO granules in F. gigantica were ovoid and were smaller and less electron-dense than those described in F. hepatica (160 X $90 \mathrm{~nm}$ in $F$. gigantica compared to $220 \mathrm{~nm}$ diameter in F. hepatica). Comparison of the tegument of newly excysted $F$. gigantica with that of in vitro penetrated juveniles indicates that the TO granules, which densely pack the perinuclear cytoplasm of the newly excysted metacercariae, move into the surface syncytium via the connecting tubules before and during in vitro penetration through mouse gut bags. The TO granules are not, at this stage, being manufactured in the perinuclear regions which, consequently, collapse and become shrunken. The space thus created in the body is taken up by expansion of the surrounding tissues, namely the parenchyma and the muscle cell bodies, to which the tegumental cells are linked by tight dense junctions between their respective bounding plasma membranes. This rapid expansion (occurs within a 5 hour period in vitro) may be achieved by uptake of fluid, since the newly appearing spaces are quite electron-lucid, and indeed, fluid uptake by the surrounding tissues may represent a mechanism for compressing the tegumental cells and helping to eject their contents towards the surface. In the tegumental syncytium, T0 granules increase in number due to upward migration from the perinuclear regions during the penetration process. The discharge of TO granules at the apical plasma membrane of the syncytium by exocytosis enables their contents to contribute to the glycocalyx that lines the outer aspect of the membrane, and is actively replaced to protect the surface of the fluke from potentially damaging interactions with the new environment, including attack by mediators of the host's immune system. The process of T0 production, migration to the surface, and exocytosis at the apical membrane in F. hepatica was described by Bennett and Threadgold (1975), and the likely significance of this process for immune protection of the migrating fluke was discussed by Hanna (1980a) and by Fairweather et al. (1999). In adult $F$. hepatica, the glycocalyx of the tegumental syncytium has been shown to comprise two zones, an inner continuous layer, $25 \mathrm{~nm}$ thick, bound to the apical plasma membrane and 
composed mainly of glycoprotein, with an outer fibrillar layer, 25-35 nm thick, comprising projecting oligosaccharide side chains though to contain terminal sialic acid residues based on its lectin-binding pattern (Threadgold, 1976). This is intriguing since helminths lack the enzymatic machinery required for sialylation and suggests that sialic acid-bearing sugars could be host-derived as a means of defence against the host immune response (Ravida et al. 2016). Lectins have been used to characterise the carbohydrate components of the glycocalyx, and the presence of mannose, glucosamine or glucose moieties and $\mathrm{N}$ acetylglucosamine was reported by Rogan and Threadgold (1984). The glycosylation of the tegumental apical membrane of adult $F$. hepatica was systematically investigated using lectin microarrays by Ravidà et al. (2016), who pointed out the likely significance of glycocalyx components as potential vaccine candidates, noting the fact that the tegumental glycocalyx is continuously exposed to the host immune system. It appears that the glycocalyx of migrating juvenile $F$. hepatica provides one of the earliest and most intense stimuli to the immune effector mechanisms of the host (Hanna, 1980 a, b; Fairweather et al., 1999), and a similar observation has been made regarding the immunogenic potential of the glycocalyx in invading juvenile F. gigantica in the bovine host (Hanna and Jura, 1977). However, evidence has yet to be adduced that the glycocalyx embodies moieties capable of engendering an immunoprotective response in the host (Hanna et al., 1988).

The most striking change in the ultrastructure of the tegument in in vitro penetrated juvenile $F$. gigantica, compared to that of the newly excysted metacercaria, is the appearance of numerous large heterogeneously-sized and -shaped electron-dense bodies which occur within the cytoplasm of the surface syncytium and the TO tegumental cells. These bodies may be compared with the secondary lysosomes and residual bodies described by Threadgold and Arme (1974) in the cytoplasm of the parenchymal cells of adult F. hepatica which had been subjected to nutritional stress in vitro, and were shown to be mobilising cytoplasmic glycogen reserves by autophagy. The mechanism involved sequestration of areas of cytoplasm containing glycogen with smooth membranous cisternae derived from mitochondria, followed by the addition of lysosomal enzymes synthesized by the GER-Golgi system. Within the autophagosomes so-formed, glygogen was hydrolysed, glucose molecules were absorbed across the limiting membrane into the cytoplasm, and unhydrolysed material remained to form electron-dense residual bodies. A similar process was described in the sustentacular tissue of adult $F$. hepatica testis by Hanna et al. (2012), which apparently serves a scavenging purpose to remove effete cells and cytoplasmic debris. In the T0 tegumental cells of $F$. gigantica, it appears that areas of cytoplasm containing clusters of T0 granules (remaining after the majority have been transported to the syncytium) are sequestered by smooth membranous sacs with electronlucid contents, possibly representing smooth endoplasmic reticulum (SER). Irregular dense granules, probably representing primary lysosomes generated by a GER-Golgi -mediated mechanism, are incorporated within the sequestered areas of cytoplasm, or are added to them subsequently by fusion of the bounding membranes, thus forming secondary lysosomes or autophagosomes. These autophagosomes, at first irregular in shape and having two or more bounding membranes, often separated by spaces of varying width, become smoother, generally oval and bounded by a single membrane due to membrane 
fusion. The process is similar that described for the parenchymal cells of adult $F$. hepatica by Threadgold and Arme (1974), although in F. gigantica T0 cells, the smooth sequestering membranous sacs are not derived from mitochondria. In juvenile $F$. gigantica, it is likely that autophagy is carried out to remove T0 granules that have not been utilised in the gutpenetration process. This may facilitate cytoplasmic reorganisation which accompanies the reactivation of biosynthetic activity and leads to production of novel products such as T1 secretory granules. TO granules are produced and stored in the TO cells at a much earlier stage, during the development of the cercaria/metacercaria (Bennett and Threadgold, 1973), and are not synthesised de novo during the excystment and penetration processes. It is likely that novel products such as T1, newly synthesised in the T0 cells once the stored T0 granules have been transferred to the syncytium or removed by autophagy, have modified physiological and possibly enzymological attributes to facilitate the next phase of host invasion, namely penetration of the liver capsule. Bennett and Threadgold (1975) noted that the TO cells in F. hepatica metamorphose and convert to biosynthesis of T1 granules after gut penetration, in preparation for liver invasion. The immunogenic properties of the new secretory products may be similar, at least in part, to the T0 granules (Hanna, $1980 \mathrm{~b}$; Hanna and Trudgett, 1983; Fairweather et al., 1999). Removal of the residual bodies resulting from autophagy in the TO cells is apparently accomplished by transport to the surface syncytium (via the trabeculae) followed by a form of apocrine secretion at the apical membrane, in which the dense body buds from the surface, invested by a narrow zone of cytoplasm. This peculiar mechanism may reflect fusion-incompatibility between the bounding membrane of the dense body and the apical plasma membrane of the syncytium. The occurrence of this unusual secretory activity, during a narrow window of the migration process, may deserve analytical scrutiny, bearing in mind that a variety of novel, potentially immunogenic components might be temporarily exposed for immune recognition by the host. The residual bodies, having originated as autophagosomes, are likely to contain lysosomal hydrolases of the cathepsin type. Indeed, silencing of cathepsin B and L proteases by RNA interference significantly reduced penetration of rat gut bags by newly-excysted $F$. hepatica metacercariae (McGonigle et al. 2008) which highlights a functional role for these enzymes during establishment in the host intestine. Accordingly, these molecules may have significance as vaccine candidates and immunomodulatory molecules (Jayaraj et al., 2010; Fairweather, 2011). In the in vitro experimental situation described here, it is possible that autophagic activity in the tegumental cells of the newly penetrated juvenile $F$. gigantica might be more intense and conspicuous that would be the case during natural infections in the ruminant host. This is because the thickness of the intestine wall in ruminants is greater than that of the mouse gut bags used. Consequently, penetration would involve burrowing through a thicker layer of tissue, with correspondingly greater expenditure of T0 secretions, and fewer TO granules might remain to be disposed of by autophagy. Consideration of the ability of the TO cells to re-organise and re-direct their various activities, including cytoplasmic storage of granules, mobilisation of secretory products to the syncytial compartment, and biosynthesis of novel components, underlines the adaptability and fundamental conservatism in the tegumental system. The single T0-type cell acts as a store for pre-formed T0 granules that are immediately available to facilitate the penetration process; undergoes lysosome-mediated autophagy; carries out GER-Golgi-linked 
biosynthesis of novel secretory products including lysosomes and T1 granules; and synthesises stores and transports actin-like crystalline spine-protein material (Stitt et al., 1992). In an earlier phase of development, the same T0 cells synthesise store and mobilise the scroll-like bodies, remnants of which were seen in both the newly excysted and in vitro penetrated $F$. gigantica. These structures comprise packaged keratin sheets which unwind and contribute to the inner wall during formation of the metacercarial cyst (Dixon, 1965; Dixon and Mercer, 1964, 1967). Finally, the swollen cytoplasmic bulb-like structures attached by junctional complexes to the basal membrane of the tegumental syncytium in both the newly excysted and in vitro penetrated F. gigantica, represent sensory receptors, of which three types (respectively tangoreceptors, pressure receptors and chemoreceptors) have been described for juvenile F. hepatica (Bennett, 1975; Fairweather et al., 1999).

\section{Conclusion}

In order to compare the ultrastructural features of the tegument of newly excysted $F$. gigantica metacercariae with those of juvenile flukes that have completed penetration through the gut of a prospective host, established in vitro procedures for excystment and penetration through mouse gut 'bags' were utilised. These two transient events in the invasion process represent the earliest exposure of vulnerable juvenile flukes to a potentially hostile physiological and immunological environment. The interaction of host immune mediators with the immunoprotective mechanisms of the invading flukes, at the interface between host and parasite, are likely to have fundamental importance for the outcome of infection and the ability of the host to mount an effective barrier to parasite establishment. Recent transcriptomics and proteomics analysis has shown that, within three hours post-excystment, $F$. hepatica newly excysted juveniles undergo significant metabolic and developmental changes, and secrete a specific collection of virulence-associated proteins in order to establish infection in the mammalian host (Cwiklinski et al. 2018). The current study suggests that ultrastructural level re-modelling of the tegument accompanies this larval activation in Fasciola spp. Clearly, antigenic moieties expressed by flukes during this ephemeral phase of the invasion process may have significance as vaccine candidates. In newly excysted $F$. gigantica metacercariae, the T0 tegumental cells are packed with preformed T0 granules, which are transported to the surface syncytium and are discharged by exocytosis at the apical surface to maintain the glycocalyx. The inner glycoprotein zone of the glycocalyx is directly apposed to the outer aspect of the apical plasma membrane, while the outer fibrillar oligosaccharide zone is in direct contact with the immunological environment of the host. The glycocalyx is turned over rapidly to prevent immune-mediated damage to the parasite surface. The TO tegumental cells shrink as their content of TO granules moves to the surface, but body shape is maintained by distention of surrounding parenchyma and muscle cell processes. The TO cells initiate autophagy to remove TO granules that were not discharged. Unhydrolysed residual material is packaged as irregularly-sized dense bodies for export to the surface syncytium and ultimately for discharge by apocrine secretion. These bodies may contain lysosome-derived molecules with potential for exploitation as vaccine candidates and immunomodulatory substances. 
Autophagy allows reorganisation of the cytoplasm for a phase of biosynthesis. The latter results in the production of $\mathrm{T} 1$ granules, which are physiologically fitted to facilitate the next phase of host invasion, namely penetration of the liver capsule. Other components seen in the fluke tegument, such as spine-protein, keratin scrolls and sensory bulbs illustrate the wide diversity of function and the adaptability of this tissue, underlining its fundamental roles in facilitating establishment of the parasite within the definitive host.

\section{Conflict of interest}

No actual or potential conflict of interest was identified that could inappropriately influence or be perceived to influence, the outcome of this work.

\section{Acknowledgements}

Our thanks are due to Mr R. Ogola and Ms E. Wairimu of the Department of Parasitology, East African Veterinary Research Organisation, Muguga, Kenya, for expert technical assistance. No external funding was obtained for this work. M.W.R was supported by a grant (BB/L019612/1) from the Biotechnology and Biological Sciences Research Council (BBSRC).

\section{References}

Bennett, C.E., 1975. Surface features, sensory structures and movement of the newly excysted juvenile Fasciola hepatica L. J. Parasitol. 61, 886-891.

Bennett, C.E., Threadgold, L.T., 1973. Electron microscope studies of Fasciola hepatica. XIII. Fine structure of newly excysted juvenile. Exp. Parasitol. 34, 85-99.

Bennett, C.E., Threadgold, L.T., 1975. Fasciola hepatica: development of tegument during migration in mouse. Exp. Parasitol. 38, 38-55.

Cwiklinski, K., Jewhurst, H., McVeigh, P., Barbour, T., Maule, A.G., Tort, J., O'Neill, S.M., Robinson, M.W., Donnelly, S., Dalton, J.P. 2018. Infection by the Helminth Parasite Fasciola 
hepatica Requires Rapid Regulation of Metabolic, Virulence, and Invasive Factors to Adjust to Its Mammalian Host. Mol. Cell. Proteomics. 17, 792-809.

Dalton, J.P., Robinson, M.W., Mulcahy, G., O’Neill, S.M., Donnelly, S., 2013.

Immunomodulatory molecules of Fasciola hepatica: candidates for both vaccine and immunotherapeutic development. Vet. Parasitol. 195, 272-285.

Dixon, K.E., 1964. Excystment of metacercariae of Fasciola hepatica L. in vitro. Nature 202, $1240-1241$.

Dixon, K.E., 1965. The structure and histochemistry of the cyst wall of the metacercaria of Fasciola hepatica L. Parasitology 55, 215-226.

Dixon, K.E., Mercer, E.H., 1964. The fine structure of the cyst wall of the metacercaria of Fasciola hepatica L. Q. J. Microsc. Sci. 105, 385-389.

Dixon, K.E., Mercer, E.H., 1967. The formation of the cyst wall of the metacercaria of Fasciola hepatica. Z. Zellforsch. Mikrosk. Anat. 77, 345-360.

Fairweather, I., 2011. Reducing the future threat from (liver) fluke: realistic prospect or quixotic fantasy? Vet. Parasitol. 180, 133-143.

Fairweather, I., Threadgold, L.T., Hanna, R.E.B., 1999. Development of Fasciola hepatica in the mammalian host. In: Dalton, J.P. (Ed.), Fasciolosis. CAB Publishing International, Oxon, pp. 47-111.

Hanna, R.E.B., 1980a. Fasciola hepatica: glycocalyx replacement in the juvenile as a possible mechanism for protection against host immunity. Exp. Parasitol. 50, 103-114. Hanna, R.E.B., 1980b. Fasciola hepatica: an immunofluorescent study of antigenic changes in the tegument during development in the rat and the sheep. Exp. Parasitol. 50, 155-170. 
Hanna, R.E.B., Jura, W., 1976. In vitro maintenance of juvenile Fasciola gigantica and their use to establish infections in mice. Res. Vet. Sci. 21, 244-246.

Hanna, R.E.B., Jura, W., 1977. Antibody response of calves to a single infection of Fasciola gigantica determined by an indirect fluorescent antibody technique. Res. Vet. Sci. 22, 339342.

Hanna, R.E.B., Baalawy, S.S., Jura, W., 1975. Methods for in vitro study of the invasive processes of Fasciola gigantica. Res. Vet. Sci. 19, 96-97.

Hanna, R.E.B., Baalawy, S.S., Jura, W., 1976. An in vitro study of the relative importance of bile and carbon dioxide in the activation of Fasciola gigantica metacercariae. Res. Vet. Sci. 20, 344-345.

Hanna, R.E.B., Trudgett, A.G., 1983. Fasciola hepatica: development of monoclonal antibodies and their use to characterise a glycocalyx antigen in migrating flukes. Parasite Immunol. 5, 409-425.

Hanna, R.E.B., Anderson, A., Trudgett, A.G., 1988. Fasciola hepatica: studies on vaccination of rats and mice with a surface antigen prepared from fluke homogenate by means of a monoclonal antibody. Res. Vet. Sci. 44, 237-241.

Hanna, R.E.B., Moffett, D., Brennan, G.P., Fairweather, I., 2012. Fasciola hepatica: a light and electron microscope study of sustentacular tissue and heterophagy in the testis. Vet. Parasitol. 187, 168-182.

Jayaraj, R., Piedrafita, D., Dynon, K., Grams, R., Spithill, T.W., Smooker, P.M., 2010. Liver fluke vaccines: vaccination against fasciolosis by a multivalent vaccine of recombinant stagespecific antigens. Procedia Vaccinol. 2, 82-85. 
MGonigle, L., Mousley, A., Marks, N.J., Brennan, G.P., Dalton, J.P., Spithill, T.W., Day, T.A., Maule, A.G., 2008. The silencing of cysteine proteases in Fasciola hepatica newly excysted juveniles using RNA interference reduces gut penetration. Int. J. Parasitol. 38, 149-155. Ravida', A., Cwiklinski, K., Aldridge, A.M., Clarke, P., Thompson, R., Gerlach, J.Q., Kilcoyne, M., Hokke, C.H., Dalton, J.P., O’Neill, S.M., 2016. Fasciola hepatica surface tegument: glycoproteins at the interface of parasite and host.

Mol. Cell Proteomics 15, 3139-3153.

Rogan, M.T., Threadgold, L.T., 1984. Fasciola hepatica: tegumental alterations as a consequence of lectin binding. Exp. Parasitol. 57, 248-260.

Spithill, T.W., Smooker, P.M., Copeman, D.B., 1999. Fasciola gigantica: epidemiology, control, immunology and molecular biology. In: Dalton, J.P. (Ed.), Fasciolosis. CAB Publishing International, Oxon, pp. 465--525.

Stitt, A.W., Fairweather, I., Trudgett, A.G., Johnston, C.F., Anderson, S.M.L., 1992. Localisation of actin in the liver fluke, Fasciola hepatica. Parasitol. Res. 78, 96-102. Threadgold, L.T., 1963. The tegument and associated structures of Fasciola hepatica. Q. J. Microsc. Sci. 104, 505-512.

Threadgold, L.T., 1967. Electron-microscope studies of Fasciola hepatica. III. Further observations on the tegument and associated structures. Parasitology 57, 633-637.

Threadgold, L. T., 1976. Fasciola hepatica: ultrastructure and histochemistry of the glycocalyx of the tegument. Exp. Parasitol. 39, 119-134.

Threadgold, L.T., Arme, C., 1974. Electron microscope studies of Fasciola hepatica. XI. Autophagy and parenchymal cell function. Exp. Parasitol. 35, 389-405. 
Turk, V., Stoka, V., Vasiljeva, O., Renko, M., Sun, T., Turk, B., Turk, D., 2012. Cysteine

cathepsins: from structure, function and regulation to new frontiers. Biochim. Biophys. Acta. $1824,68-88$.

Walker, S.M., Prodöhl, P.A., Hoey, E.M., Fairweather, I., Hanna, R.E.B., Brennan, G., Trudgett, A., 2012. Substantial genetic divergence between morphologically indistinguishable populations of Fasciola suggests the possibility of cryptic speciation. Int. J. Parasitol. 42, 1193-1199.

\section{Legends for figures}

Fig. 1. (a-c) Electron micrographs of Fasciola gigantica, newly excysted metacercariae. (a) The tegument comprises an anucleate surface syncytium (S), bounded by a deeply invaginated apical plasma membrane (am) and an undulating basal plasma membrane (bm). The syncytium is connected by vacuolated trabeculae (tb) to a nucleated zone or 'tegumental cells' (TC), which lies beneath the myofibrils (M) of the superficial muscle layer. Each tegumental cell bears a heterochromatic nucleus $(N)$ with a single nucleolus (nu), while the nuclei of embryonic cells ( $\mathrm{Ne}$ ) and of muscle cells $(\mathrm{Nm})$ appear much more electrondense. Lipid droplets (Li) are evident in the parenchyma, and smaller droplets appear in the tegumental cell cytoplasm (arrow). (b) Within the surface syncytium (S), elongated conical spines (Sp) are embedded. The base of each spine is anchored to the basal membrane (bm) by a dense junction (arrow), and the apical membrane (am) is continuous over the tip. The syncytium contains dense mitochondria $(\mathrm{m})$ and scattered oval T0 secretory granules (TO). Beneath the muscle layers (M), tegumental cell bodies (TC) are densely packed with TO granules, and dense mitochondria, similar to those in the syncytium, are abundant. (c) Higher magnification of a spine (Sp) in the surface syncytium reveals its striated appearance, and the base is anchored to the basal membrane of the syncytium by a dense junction (arrow). Immediately beneath is the basal lamina (bl).

Fig. 2. (a-d) Electron micrographs of Fasciola gigantica, newly excysted metacercariae. (a) In the surface syncytium, T0 secretory granules (TO) are seen close to the apical plasma membrane (am), which has a 'fuzzy' appearance. Occasionally a T0 granules is seen to discharge its content by exocytosis at the surface (arrow), leaving the limiting membrane of the granule as a flattened membranous sac (TOf) in the apical cytoplasm. Sp = spine. (b) Flattened membranous sacs (TOf) of discharged TO granules are abundant in the distal 
cytoplasm, whereas intact T0 granules (TO) tend to predominate deeper in the syncytium. M = muscle myofibrils; $\mathrm{Sp}=$ spine; $\mathrm{tb}=$ trabeculum; $\mathrm{SB}=$ sensory ending; $\mathrm{Li}=$ lipid droplet. (c) The cytoplasm of the tegumental cells is packed with stored T0 secretory granules (TO), while ribosomes and polyribosomes $(r)$ tend to be concentrated close to the nucleus $(N)$ and at the periphery. Dense mitochondria $(\mathrm{m})$ are numerous, and occasional strands of granular endoplasmic reticulum are seen (Ger). Golgi bodies with stacked lamellae (Go) are prominent, and occasional dense spherical secretory granules (T1), approximately $80 \mathrm{~nm}$ in diameter, are sometimes seen near the maturing face. $M=$ muscle. (d) Golgi bodies (Go) sometimes have dense secretory granules (T1) in the vicinity of the maturing face. $N=$ nucleus; $m$ = mitochondrion; $r$ = ribosomes and polyribosomes; $\mathrm{T0}=\mathrm{T0}$ secretory granules; Ger = granular endoplasmic reticulum; $\mathrm{Li}=$ lipid droplet.

Fig. 3. (a-d) Electron micrographs of Fasciola gigantica, newly excysted metacercariae. (e) Electron micrograph of Fasciola gigantica, in vitro penetrated juvenile. (a) Non-membranebound mass of spine material (Sp) in the cytoplasm of a tegumental cell. (b) Profile of a scroll-like body $(\mathrm{Scr})$ in the cytoplasm of a tegumental cell. T0 = T0 secretory granules; $\mathrm{m}=$ mitochondrion. (c) High magnification of a dense trilaminate membrane junction (arrow) between a tegumental cell, that contains TO secretory granules (TO) and mitochondria (m) and a parenchymal cell extension (P). (d) An irregular vacuolated trabeculum (tb) penetrates between myofibrils (M) to enter the base of the surface syncytium (S). A cluster of T0 secretory granules (TO) is present in the lumen of the trabeculum. $\mathrm{Nm}=$ nucleus of muscle cell. (e) Low power micrograph showing numerous dense bodies (Db) in the cytoplasm of the surface syncytium (S) and in the tegumental cells, often close to the nuclei $(N)$. Dense bodies bulge from the tegumental syncytium in all regions of the body, including the lining of the acetabulum (A), and are occasionally seen budding from the surface (arrows).

Fig. 4. (a-d) Electron micrograph of Fasciola gigantica, in vitro penetrated juvenile. (a) In the surface syncytium (S) T0 secretory granules (TO) are particularly abundant and spines (Sp) are frequent. Mitochondria $(\mathrm{m})$ often appear swollen and electron-lucid, and dense bodies (Db) are present in the cytoplasm. $M=$ myofibrils of subsyncytial musculature. (b) T0 granules (TO) are very abundant in the syncytial cytoplasm, but in some areas, smaller electron-dense T1 secretory granules (approximately 80nm in diameter) (T1) are irregularly distributed. Flattened membranous sacs representing discharged T0 granules (TOf) are most abundant beneath the apical plasma membrane, which typically has a rather 'fuzzy' appearance (arrow). DB = dense body; $m$ = mitochondrion. (c) Beneath the surface syncytium $(\mathrm{S})$ and anchored to the basal membrane $(\mathrm{bm})$ by a junctional complex, bulbous sensory endings (SB) contain variously-sized vesicles. $\mathrm{Db}=$ dense body. (d) The tegumental cells (TC) are shrunken and contracted, with corresponding distention and vacuolation of the surrounding parenchymal tissue $(P)$. Dense bodies $(\mathrm{Db})$ are seen in the cytoplasm of the tegumental cells, often close to the nuclei $(\mathrm{N})$, and in the surface syncytium (S). Clusters of TO secretory bodies are partially sequestered by membranous sacs (arrow). $M=$ muscle; $m=$ mitochondria.

Fig. 5. (a-d) Electron micrograph of Fasciola gigantica, in vitro penetrated juvenile. (a) Close to the nucleus $(\mathrm{N})$ of a tegumental cell, there is a fully-formed dense body (Db), and a 
cluster of T0 secretory granules (TO), together with material of irregular density, which is partially sequestered by membranous sacs (arrow). Free T0 granules are also present in the cytoplasm, and amongst these, dense lysosome-like bodies, 150-200 nm in diameter, are visible (L). Mitochondria ( $\mathrm{m}$ ) are rather swollen. (b) Dense bodies (Db) in the cytoplasm of a tegumental cell are seen apparently engulfing (arrows) T0 secretory granules, which also indent the surface of the forming bodies. $\mathrm{P}=$ parenchyma; $\mathrm{M}=$ muscle; $\mathrm{S}=$ surface syncytium. (c) Forming dense bodies (Db), lysosome-like bodies (L) and T0 secretory granules lie together in an area of tegumental cell cytoplasm that is partially sequestered by membranous sacs (arrow). Granular endoplasmic reticulum (Ger) and ribosomes surround the area. (d) Large and smaller dense bodies ( $\mathrm{Db}$ ) are seen merging in an area of tegumental cell cytoplasm that also contains TO secretory granules (TO) and lysosome-like bodies (L). Granular endoplasmic reticulum (Ger) ribosomes and mitochondria $(\mathrm{m})$ are closer to the nucleus (N).

Fig. 6. (a-d) Electron micrograph of Fasciola gigantica, in vitro penetrated juvenile. (a) High magnification of the periphery of a fully-formed dense body (Db) in the tegumental cell cytoplasm reveals it to be limited by a plasma membrane, featuring unusual densification of the bilayer (arrow). The membrane is generally single, but with traces of additional layers in places. (b) The periphery of a dense body ( $\mathrm{Db}$ ) in tegumental cell cytoplasm exhibits multiple layers of bounding plasma membranes, with dense material interspersed between the layers (arrows). (c) Two merging dense bodies ( $\mathrm{Db}$ ) in tegumental cell cytoplasm are bounded by a dense plasma membrane (black arrows). This becomes multi-layered in one area (white arrow) due to fusion with a flattened dense membranous sac. T0 secretory granules (TO) surround the dense bodies. (d) High magnification of a portion of a scroll-like body $(\mathrm{Scr})$ in tegumental cell cytoplasm. 
Figure 1

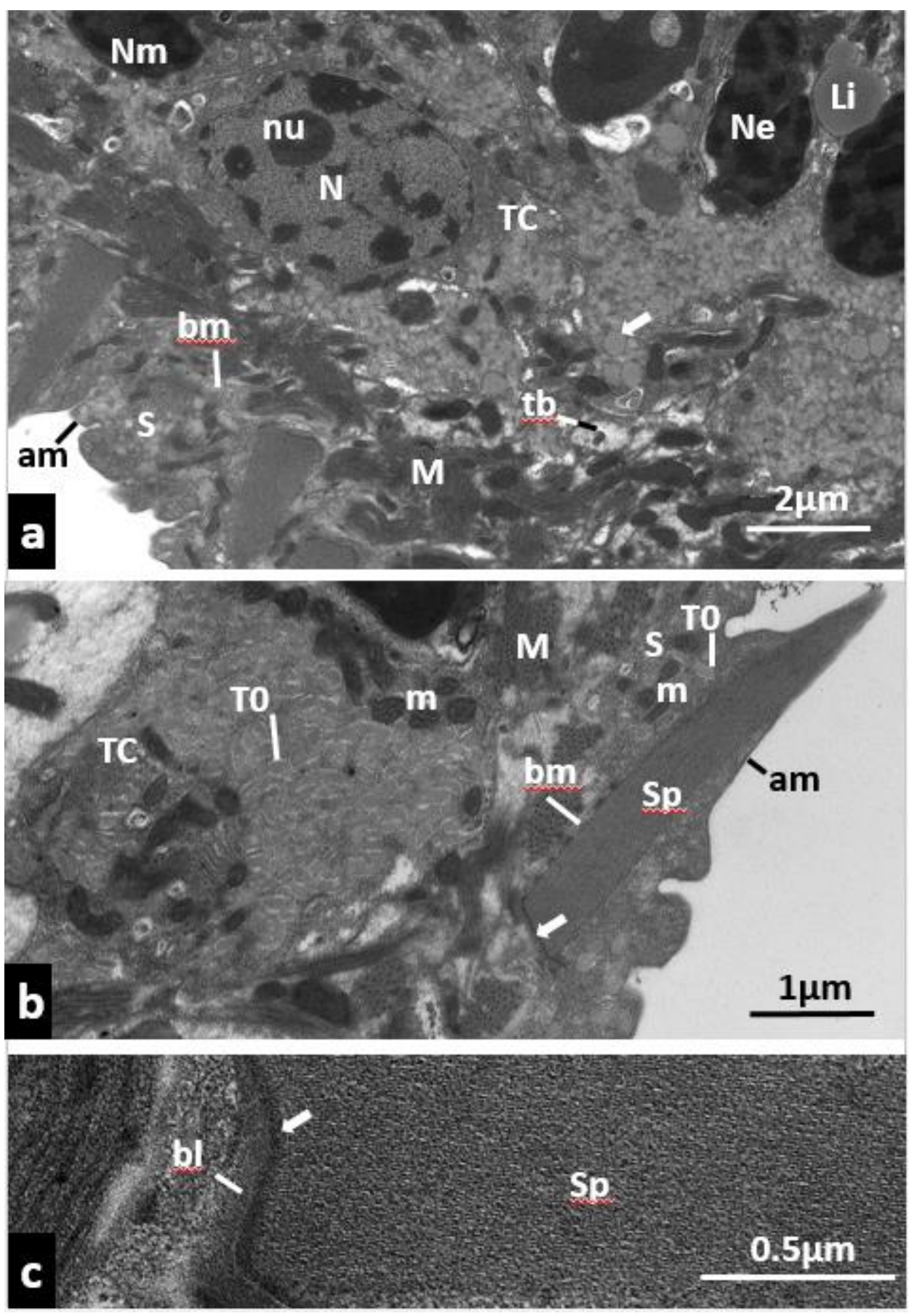


Figure 2
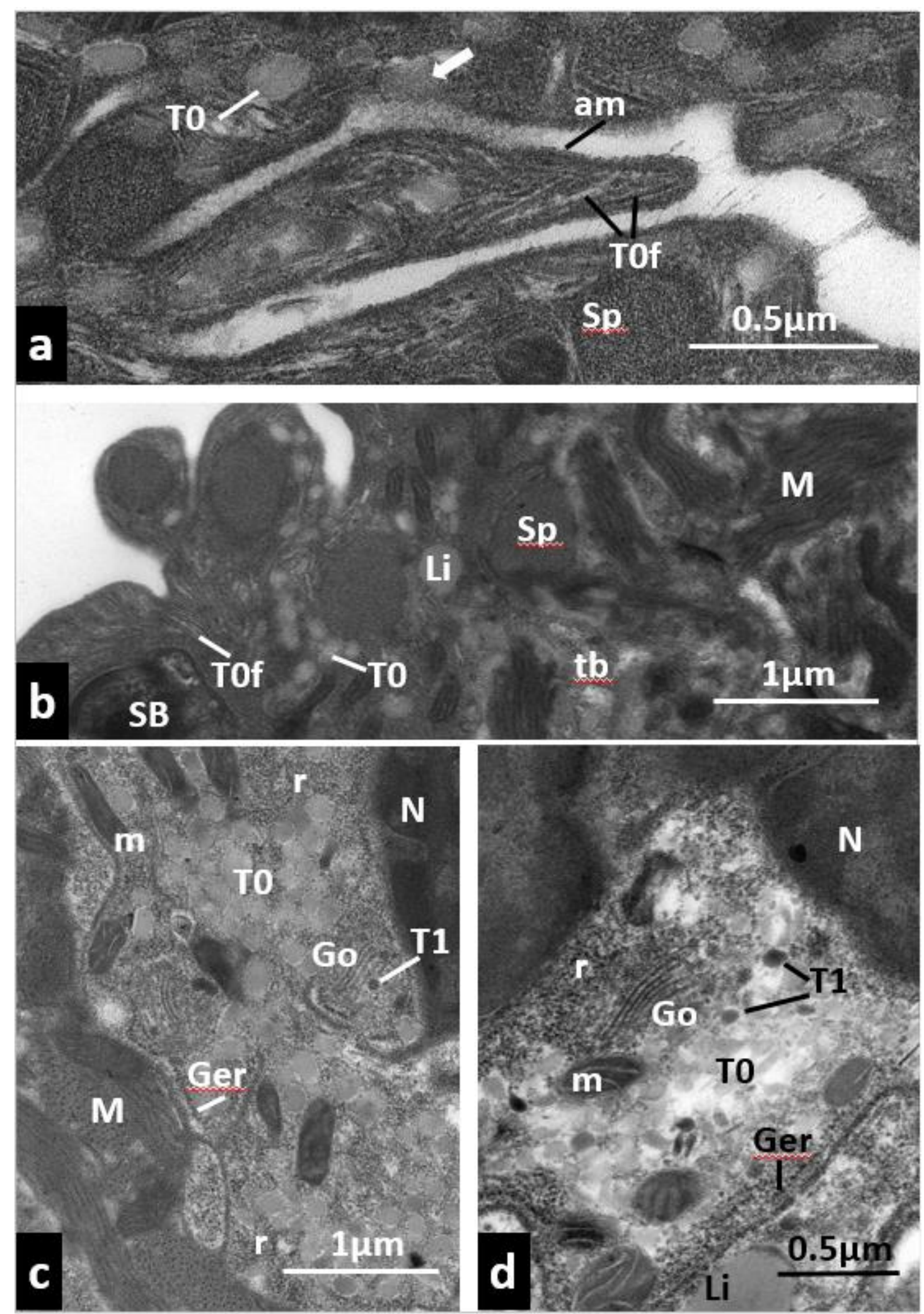
Figure 3
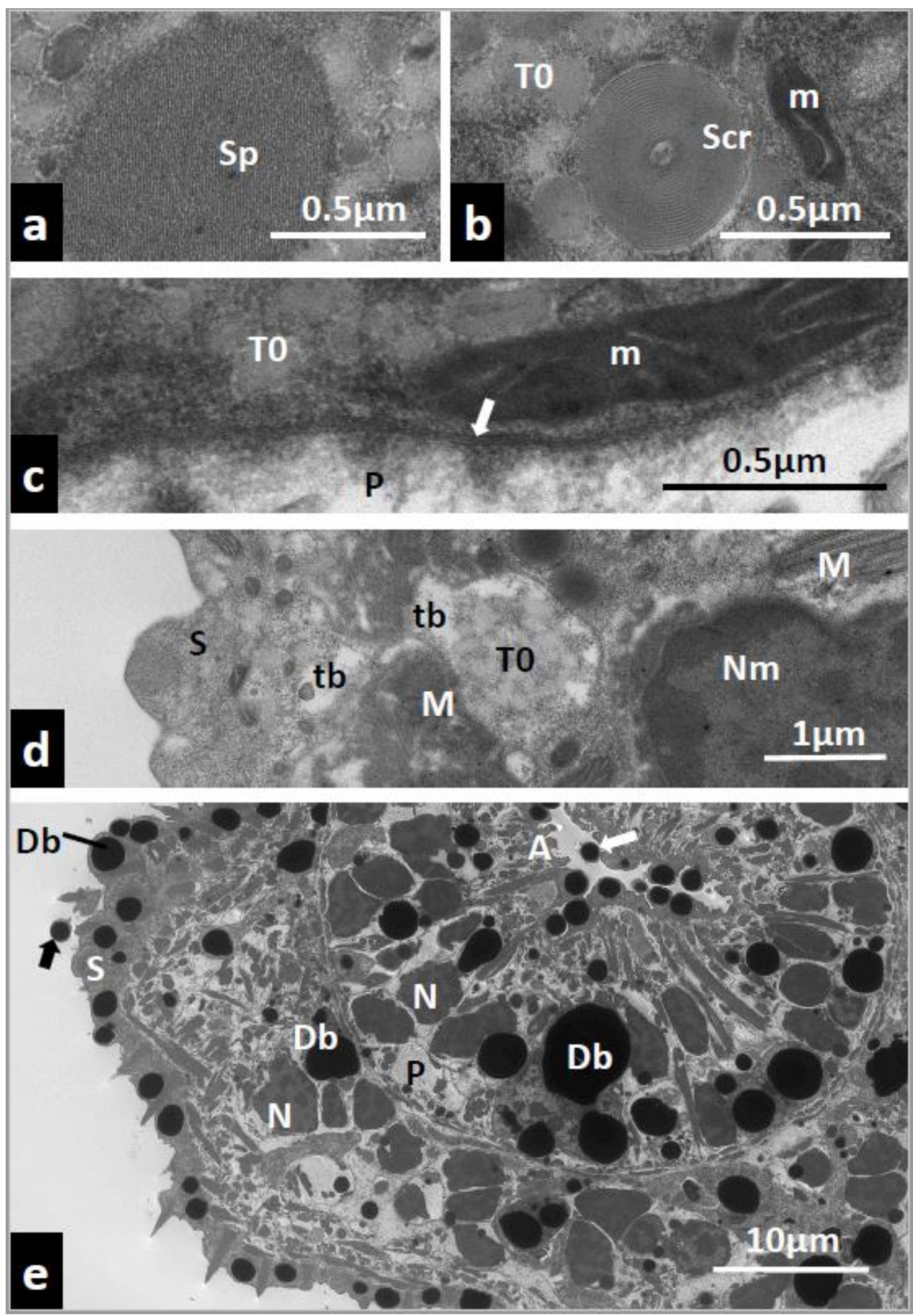
Figure 4
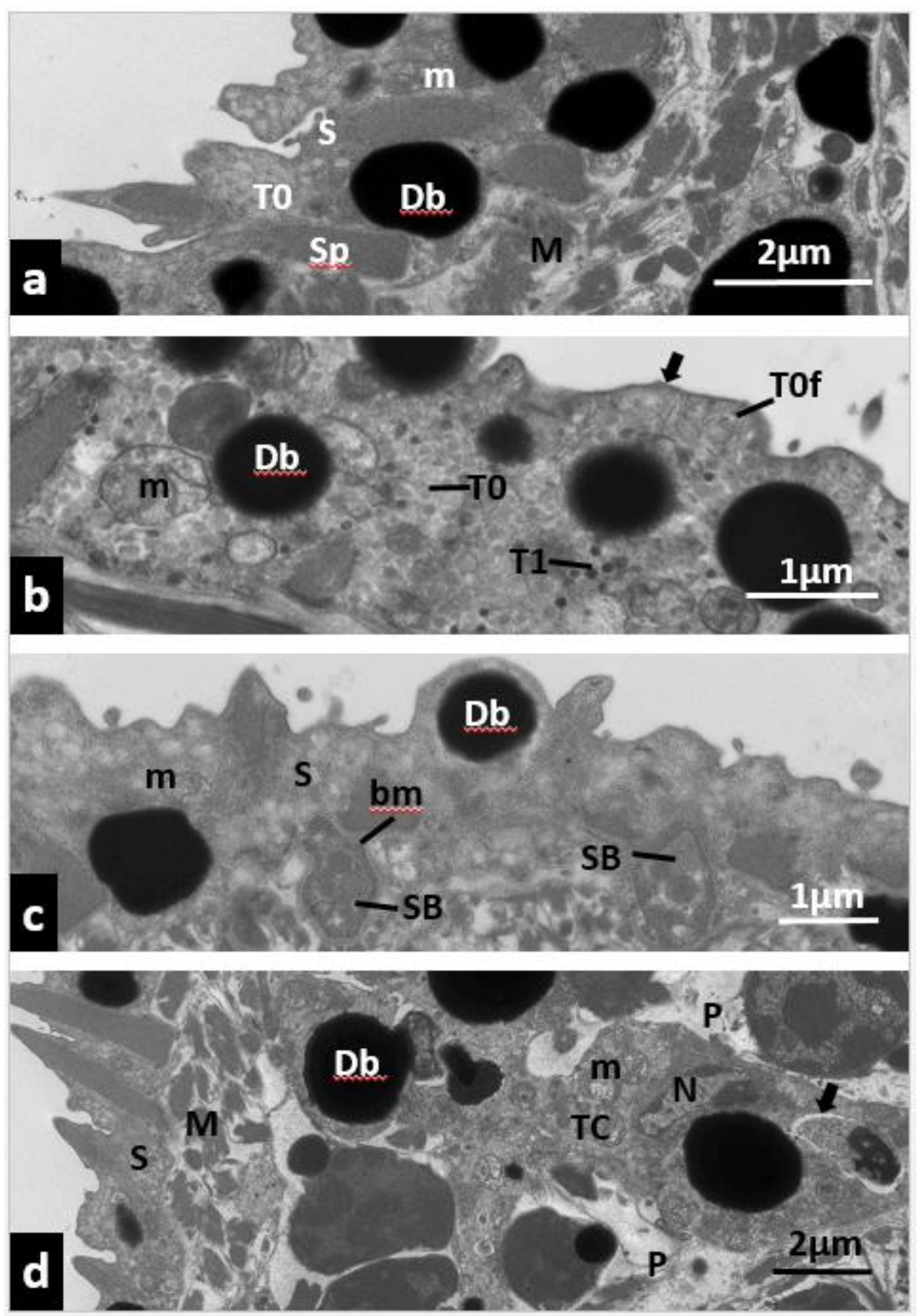
Figure 5
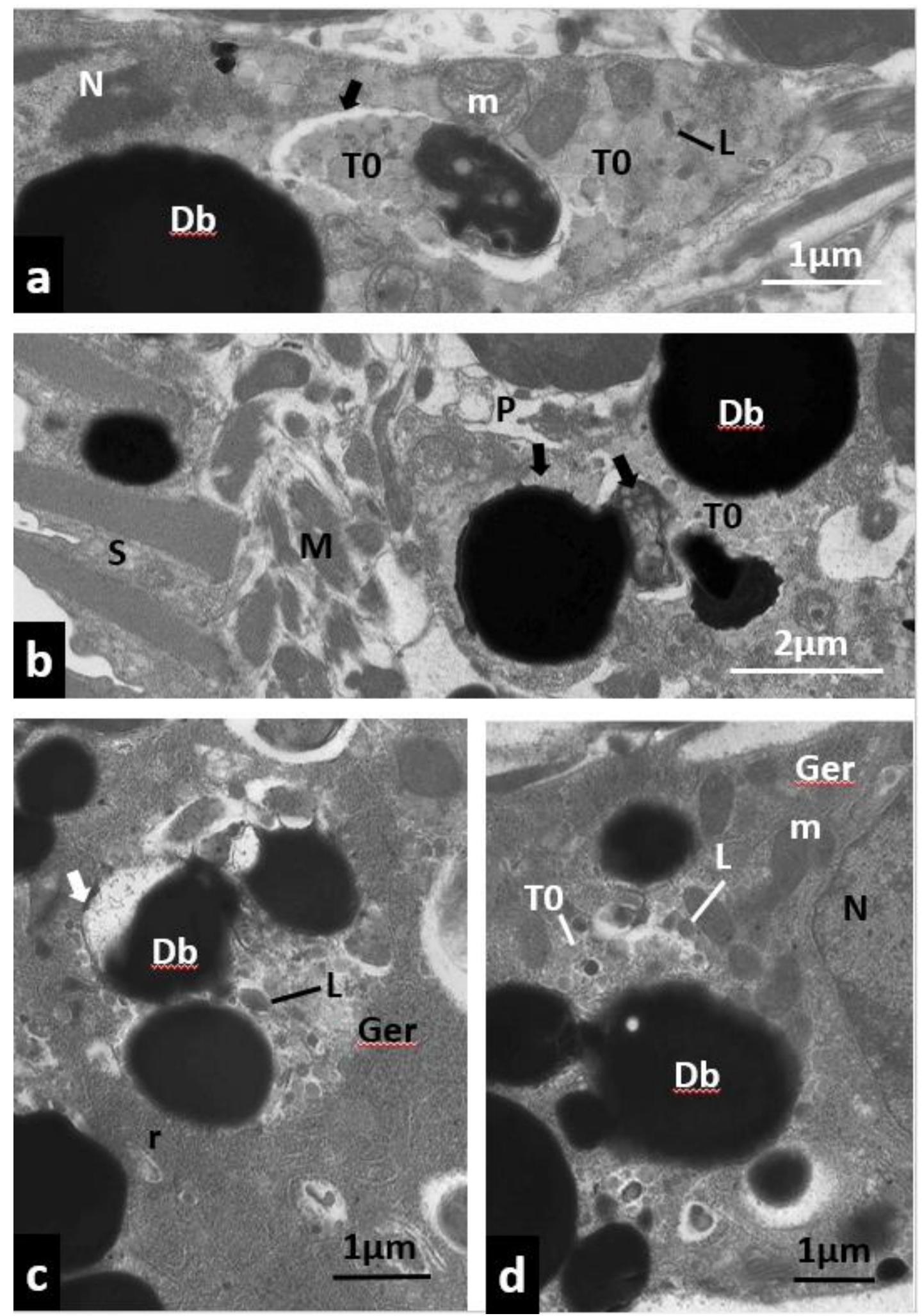
Figure 6
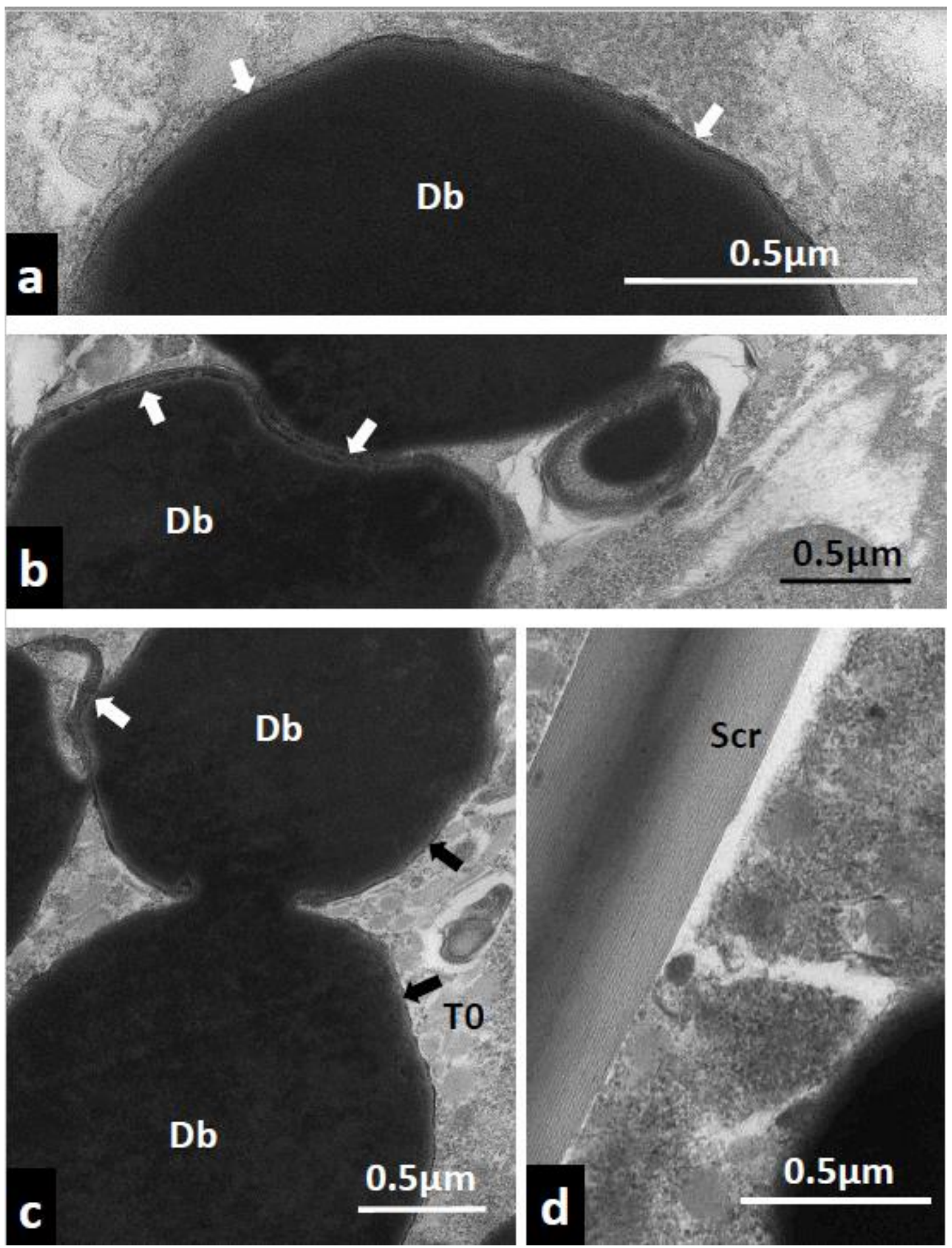